\title{
A Tunable Wavelength Demultiplexer Using Logarithmic Filter Chains
}

\author{
Cedric F. Lam, Student Member, IEEE, Rutger B. Vrijen, Member, OSA, Patty P. L. Chang-Chien, \\ Daniel F. Sievenpiper, Student Member, IEEE, and Eli Yablonovitch, Fellow, IEEE, Fellow, OSA
}

\begin{abstract}
This paper proposes a novel wavelength division multiplexer (WDM) demultiplexer design for dense WDM networks. The proposed demultiplexer consists of rectangular shaped periodic frequency filters connected in series. These rectangular filters can be formed with an apodized onedimensional (1-D) photonic crystal structure on a ridged semiconductor waveguide. A design example is given for such filters. In such designs, we can use a moderate refractive index contrast, in discrete groups of many layers, to achieve the same stop-band width that we would get with a large contrast. Apart from being compact in size, to demultiplex an arbitrary wavelength from $N$ wavelengths, only $\log _{2} N$ switches and filter stages are required. This type of filter has a large potential to be used in integrated photonic implementation and packet switched dense WDM applications.
\end{abstract}

Index Terms-Logarithmic scaling, photonic crystal, photonic switching, waveguide filter, WDM demultiplexer.

\section{INTRODUCTION}

O PTICAL fibers have $25 \mathrm{THz}$ available bandwidth in each of the three low loss operation windows [1]. In order to fully utilize the available bandwidth in fiber channels and at the same time overcome the difficulties of ultrafast electronics and fiber dispersion problems, dense wavelength division multiplexed (DWDM) systems time-shared among a large number of access nodes might be the solution for future high-bandwidth tera-bit multimedia and packet switched optical networks.

The ability to select an arbitrary wavelength in a DWDM network in a fraction of the packet duration is essential in realizing high-speed packet switched networks [2]. A lot of work on tunable wavelength filters has been done in the past. Most of the tunable wavelength filters are based on variable cavity length Fabry-Perot (FP) filter principles [3]-[5]. They are made tunable either by changing the physical distance between FP mirrors either mechanically or thermally, which is slow or using the electro-optic effect to change the refractive index between the mirrors which suffers from a small tuning range.

Wavelength demultiplexing can also be achieved using grating demultiplexers. This has been achieved using ruled

Manuscript received February 11, 1998; revised April 29, 1998.

C. F. Lam, R. B. Vrijen, D. F. Sievenpiper, and E. Yablonovitch are with the Electrical Engineering Department, University of California at Los Angeles (UCLA), Los Angeles, CA 90095 USA.

P. P. L. Chang-Chien is with the University of Michigan, Ann Arbor MI 48109 USA.

Publisher Item Identifier S 0733-8724(98)06679-1. gratings in a Stimax configuration [6] or integrated silicon waveguides using a Dragone configuration [7]. WDM filters are also made of cascaded narrow band microcavity optical filters. In all theses structures, the multiplexed signal is demultiplexed into $N$ individual wavelengths first and the desired wavelength channel is selected with an array of $N$ switches [8]. The complexity increases linearly as the number of wavelengths $N$. Since switches are active components, such a linear scaling is undesirable in a DWDM system where $N$ is large.

In Section II, we describe the principle of a wavelength demultiplexer using the bisection method to achieve $\log _{2} N$ scaling complexity for $N$ wavelengths. A proposed photonic crystal waveguide structure to achieve the filter function is described in Section III along with the simulation results. Section IV describes a few possible settings in which the proposed waveguide can be incorporated for switching purposes. Section V concludes this paper.

\section{PRINCIPLE}

To achieve the desired $\log _{2} N$ scaling, we can arrange a series of filters in a way that half of the spectral energy is filtered out when the input spectrum is passed through each successive filter stage. Each filter stage has a rectangular shaped periodic frequency transfer function. The first filter stage has the smallest period, or free spectral range. The filter of the next stage has twice the periodicity of the previous stage. So the first stage filters out half of the spectrum. The second stage filters out another half of the spectrum that is left from the output of the first stage, etc. Optical filters are usually interferometric devices which generate periodic complementary output at the same time. An example is a Mach-Zehnder interferometer (MZI). Fig. 1(a) shows the spectrum of an eight-wavelength WDM signal. Fig. 1(b) shows the filter function of each stage in a three-stage cascaded eight-wavelength demultiplexer. The solid lines and dashed lines represent the direct and complementary response of each filter stage. Each filter stage which produces complementary spectral outputs is followed by a $2 \times 2$ optical switch. The control signals at the $2 \times 2$ optical switches select either the direct or the complementary output of each filter stage. By properly combining the direct or complementary transfer function of each stage, we are able to select any one wavelength in the signal spectrum shown in Fig. 1(a). Scaling now becomes $\log _{2} N$ instead of $N$, which is much 


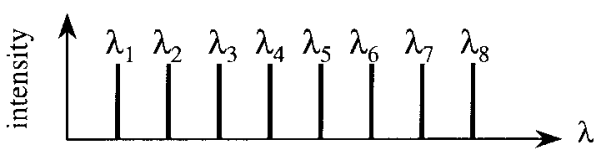

(a)

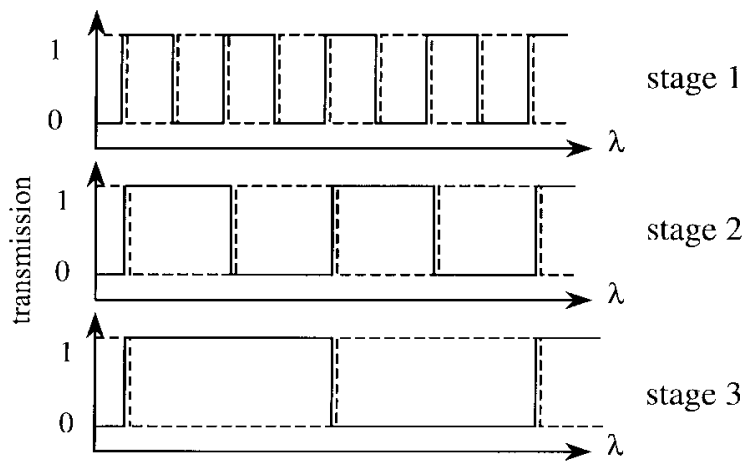

(b)

Fig. 1. (a) An eight-wavelength multiplexed WDM signal spectrum example. (b) An example of a three-stage WDM demultiplexer with the ability to isolate an arbitrary wavelength out of the eight multiplexed wavelengths in (a). The solid lines and dash lines represent the direct and complementary spectral output at each stage.

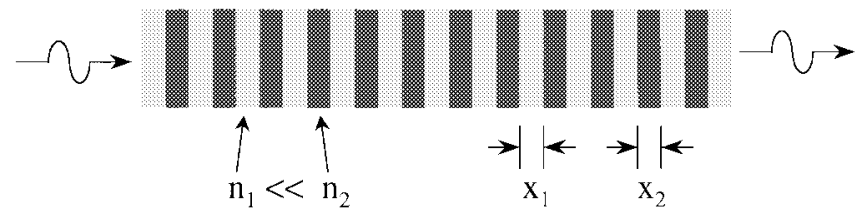

Fig. 2. A simple photonic crystal consists of alternate layers of dielectric materials. To produce equal width passband and stopband, this system requires a refractive index contrast of 5.83 .

more favorable. To select one wavelength out of 1000, only ten switches are required.

The next section discusses the design of filters which have the desired rectangular shaped periodic frequency transfer functions. These filters are based on a one-dimensional (1-D) photonic crystal structure.

\section{Photonic Crystal Filter Design}

\section{A. Simple 1-D Photonic Crystal}

To produce repeated passbands and stopbands in the frequency domain, a 1-D photonic crystal structure is used [9]. A simple 1-D photonic crystal consists of alternate layers of dielectric materials with refractive indexes $n_{1}$ and $n_{2}$ (Fig. 2). It is well-known that such periodic structures will produce periodic stopbands and passbands in the frequency domain [10]. The transition sharpness of the band edges is determined by the number of alternate layers used in the structure. The more layers used, the faster is the roll-off at the band edge.

The period of the structure $\left(n_{1} x_{1}+n_{2} x_{2}\right)$ determines the repetition frequency $\Delta f$ of the band structure and is given by $x_{i}=c /\left(2 n_{i} \Delta f\right)$ where $c$ is the speed of light in vacuum. The center frequencies $\omega=2 \pi f=\omega_{m}$ of the forbidden bands

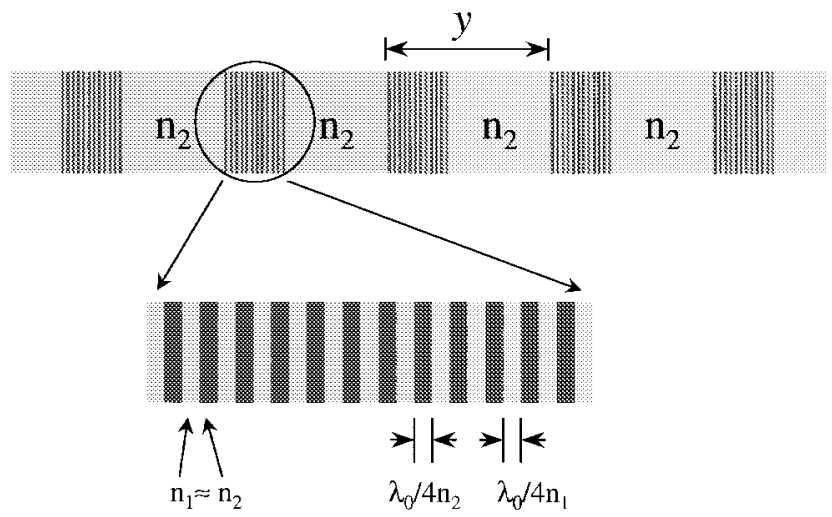

Fig. 3. The top view of a periodic rectangular shaped frequency filter that consists of effective Bragg Mirrors separated apart by distances $y$ determined by the required free spectral range. The difference between the refractive indexes $n_{1}$ and $n_{2}$ is $8.5 \%$.

occur at $[10]$

$$
k_{1} x_{1}=k_{2} x_{2}=\frac{(2 m+1)}{2} \pi \quad m=0,1,2 \cdots
$$

where $k_{i}=(\omega / c) n_{i}$. The bandgap $\Delta \omega_{\text {gap }}$ and the first band center $\omega_{0}$ is related by [10]

$$
\Delta \omega_{\text {gap }}=\omega_{0} \frac{4}{\pi} \sin ^{-1} \frac{\left|n_{2}-n_{1}\right|}{n_{2}+n_{1}} .
$$

The key to logarithmic scaling is that the stopband and passband have equal bandwidth. This condition is satisfied when $\Delta \omega_{\text {gap }}=\omega_{0}$. Substituting into (2), we obtain $n_{2} / n_{1}=$ 5.83 . This actually corresponds to a $50 \%$ power reflection at each dielectric interface. However, such a high refractive index contrast is impractical for real physical systems.

\section{B. Photonic Crystals with Effective Bragg Mirrors as Reflectors}

As we have seen before, equal width transmission band and forbidden band are obtained with periodic 50\% power reflectors separated by distances determined by the filter periodicity in the frequency domain.

Since it is difficult to achieve the high reflectivity required with a single reflecting surface, as proved in the last section, we propose to use Bragg mirrors consisting of layers of quarter-wave dielectric materials as the reflectors in our rectangular shaped frequency filter. Fig. 3 shows the top view of such an arrangement. The refractive index and the number of layers in a Bragg mirror are chosen such that each effective mirror has a reflectivity of about $50 \%$ in the optical frequency range of interest. The best erbium doped fiber amplifier (EDFA) in today's technology covers a $80 \mathrm{~nm}$ bandwidth around the $1.55 \mu \mathrm{m}$ communication wavelength [11]. This is approximately a 5\% window around the 1.55 $\mu \mathrm{m}$ center wavelength. In order for the mirror to be effective over such a wavelength window, the number of layers has to be limited to achieve a low $Q$. On the other hand, it is easier to 

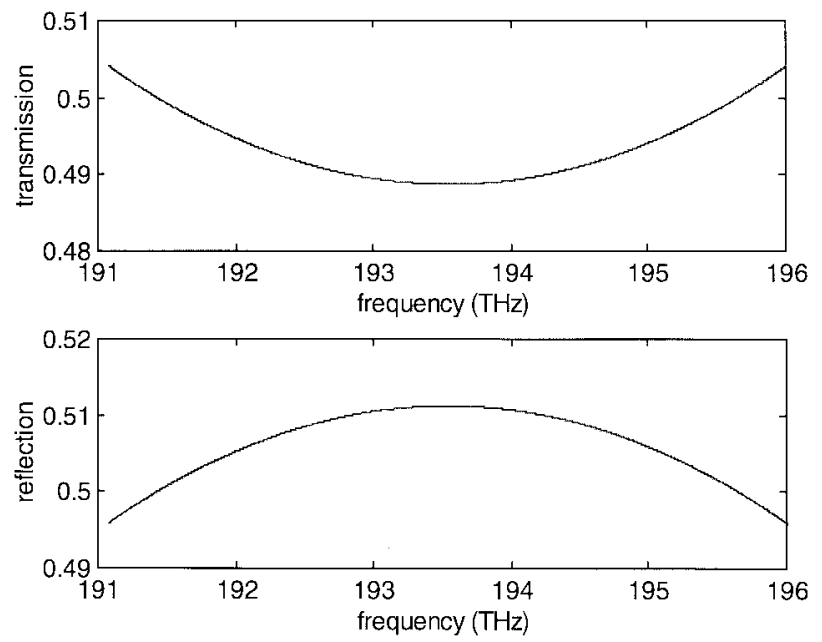

Fig. 4. The simulated transmission and reflection of the effective Bragg mirror used in the Fig. 3. Eleven periods of quarter-wave stacks are used in the simulation and the result obtained corresponds to a 40-nm wide region around the $1.55-\mu \mathrm{m}$ center wavelength.

achieve $50 \%$ reflectivity at the center wavelength with lower refractive index contrast when more layers are used.

In our design, we used a refractive index contrast of $\left(n_{2}-\right.$ $\left.n_{1}\right) / n_{1}=8.5 \%$ and eleven periods of quarter-wavelength layers. The transmission and reflection of such a periodic Bragg mirror are calculated using the transfer matrix method [10] and are plotted in Fig. 4. The input optical signal has a normal incidence on the mirror. The reflectivity is about $50 \%$ in the frequency range of interest.

Our rectangular shaped frequency transfer filter is formed by arranging these 50\% effective mirrors in series as in Fig. 3. Again, the sharpness of the band edge is determined by the number of mirrors used. The separation between the mirrors is given by

$$
y=\frac{c}{2 n_{2} \Delta f}
$$

where $\Delta f$ is the desired periodicity in the frequency domain.

By using 19 mirrors, calculated with the transfer matrix method [10], we obtained the transmission and reflection spectra shown in Fig. 5. The filter response is simulated over a $40-\mathrm{nm}$ region around the $1.55 \mu \mathrm{m}$ center wavelength. Four frequency band periods are included in this simulation. Again, we assumed normal incidence of the input optical signal to the filter. The passband and stopband have equal bandwidth and the stopband has almost complete attenuation of the input signal. Therefore, we can achieve the same stop-band width that we would get with a large index contrast, by using a moderate $\Delta n$, in discrete groups of many layers.

\section{Apodization of Bragg Mirrors}

It is seen in the above design that the filter response in the passband is far from desired. The fringes in the passband correspond to the reflections at the two end mirrors which have abrupt termination to the surroundings. In order to
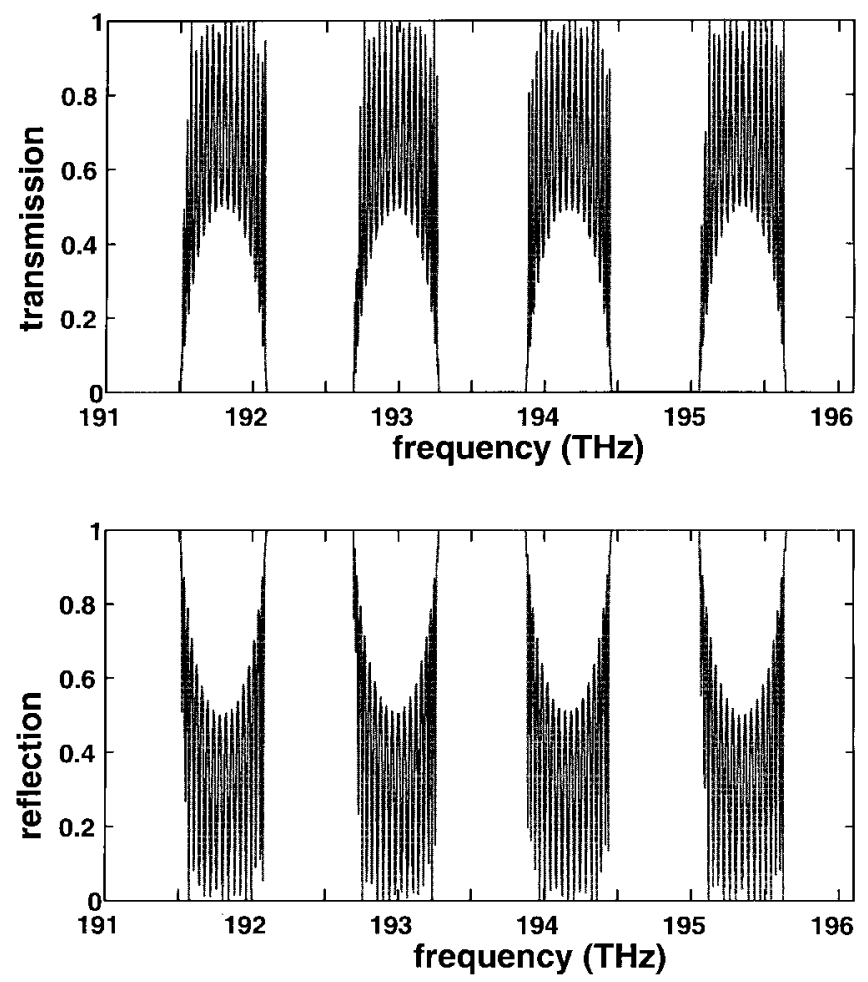

Fig. 5. The simulated transmission and reflection of the filter in Fig. 3 over a $40-\mathrm{nm}$ wide region around the $1.55-\mu \mathrm{m}$ center wavelength. Nineteen effective Bragg mirrors are separated by distances which will produce four periods in the simulated frequency range. The fringes in the passbands are due to the reflections at the two end mirrors.

"smooth" out the passband, we apodized the mirrors so that the reflectivity gradually decreases as we move from the center mirror toward the two end mirrors. This is done by decreasing the number of periods used in the Bragg mirrors using a Gaussian function rounded to the nearest integer according to the following formula:

$$
M_{i}=\left\lceil M_{(K+1 / 2)} \exp \left[-\left(\frac{i-(K+1) / 2}{\alpha}\right)^{2}\right]+0.5\right\rceil
$$

where $M_{i}$ is the number of layers used in the $i$ th mirror and $\alpha$ determines the width of the Gaussian function. The total number of mirrors $K$ is assumed to be an odd number for simplicity. We used $K=19, M_{(K+1) / 2}=11$ and $\alpha=6$ in one of our designs. Using this design, the outermost mirrors will only have one layer. The simulation result is plotted in Fig. 6. It can be seen that the passband ripple is very small.

\section{Physical Implementation of Rectangular Shaped Frequency Filters}

Fig. 7 shows the perspective view of a possible physical implementation of the apodized rectangular-shaped filter on a ridged waveguide [12]-[15] made out of common semiconductor materials. The low index of refraction dielectric could be formed, for example, by etching grooves on the ridged waveguide. Taking Si $\left(n=n_{2}=3.44\right)$ as the waveguide 

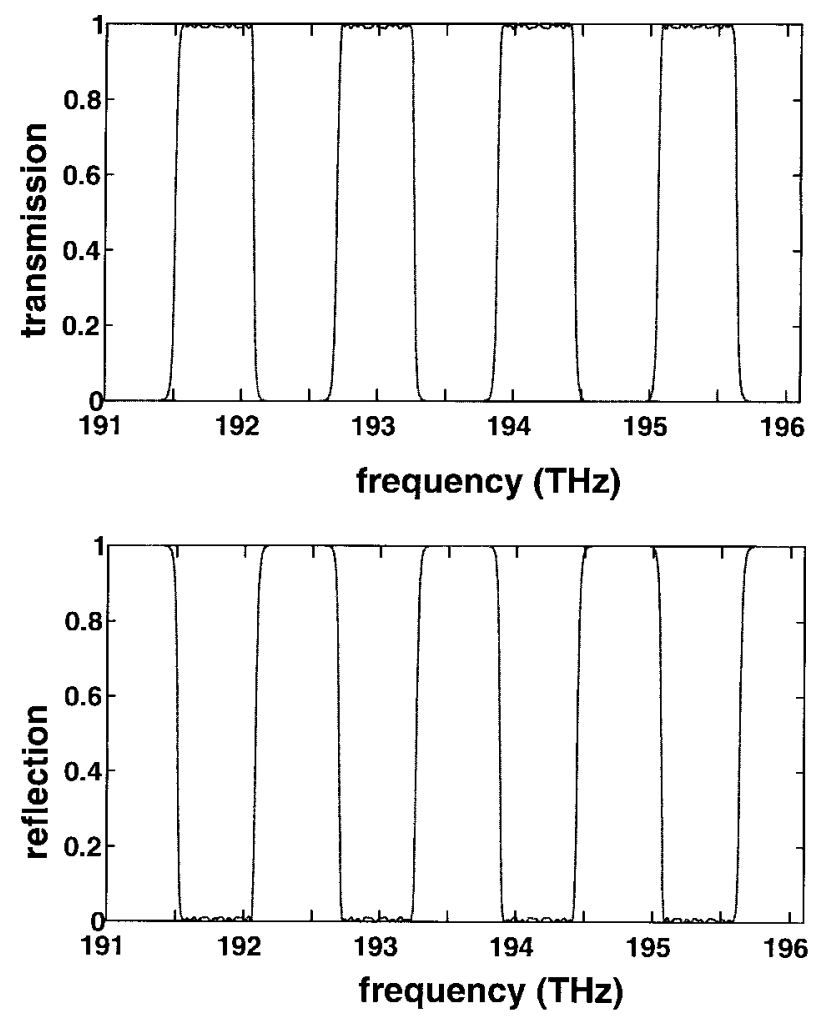

Fig. 6. The simulated transmission and reflection of a Gaussian apodized filter. The passband ripples are suppressed by the apodization.

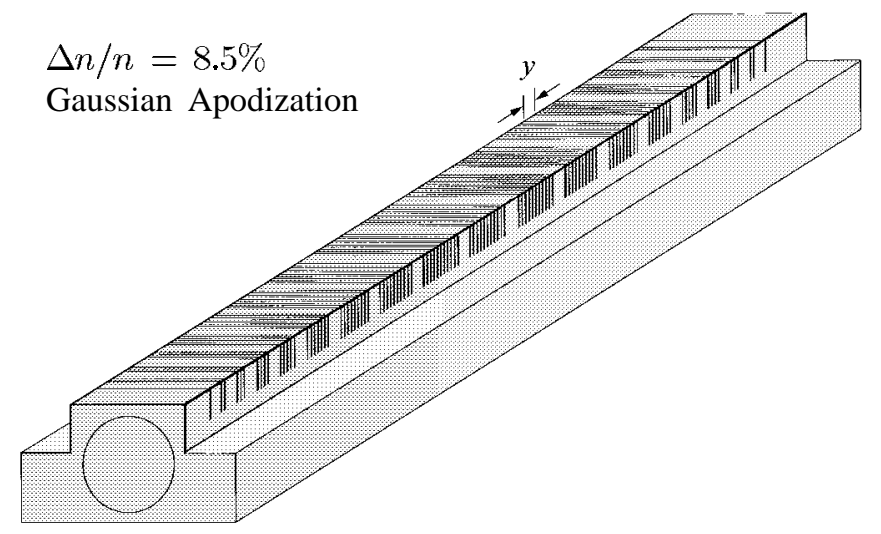

Fig. 7. The perspective view of an apodized periodic rectangular-shaped frequency filter in a ridged waveguide structure. The Bragg reflectors with apodized reflectivity are formed by etching grooves in the ridged waveguide. Each groove is a quarter wavelength $\left(\lambda_{0} / 4 n_{1}\right)$ thick and the separation between adjacent grooves in a Bragg reflector is also a quarter wavelength $\left(\lambda_{0} / 4 n_{2}\right)$ where $\lambda_{0}$ is the center wavelength of the frequency band covered. The distance between two adjacent Bragg reflectors is chosen as $y=c /\left(2 n_{2} \Delta f\right)$ in the simulation. This distance determines the free spectral range (FSR) of the filter. This figure shows the number of layers in each Bragg reflector as prescribed by (4) and as used in the numerical simulation.

substrate, each quarter-wave layer is about $0.11 \mu \mathrm{m}$. In our simulated design, the frequency domain period is $\Delta f=1.25$ $\mathrm{THz}(\sim 10 \mathrm{~nm})$ which corresponds to a mirror separation of $34.9 \mu \mathrm{m}$. Using the data that we have collected, the total length of the filter example in this design is about $654 \mu \mathrm{m}$. For the coarse stage, a larger number of mirrors needs to be used to get sharper edges. But the separation between the mirrors

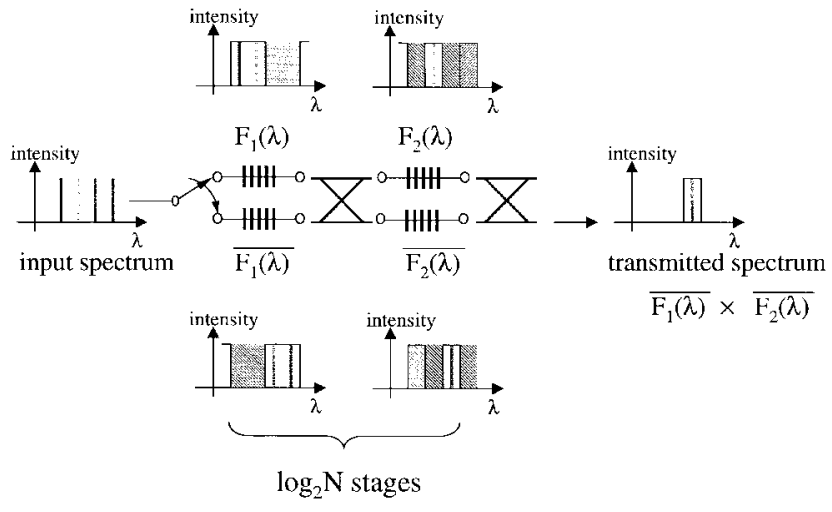

Fig. 8. Wavelength selection using $2 \times 2$ optical switches and cascaded complementary periodic rectangular-shaped spectral filter pairs.

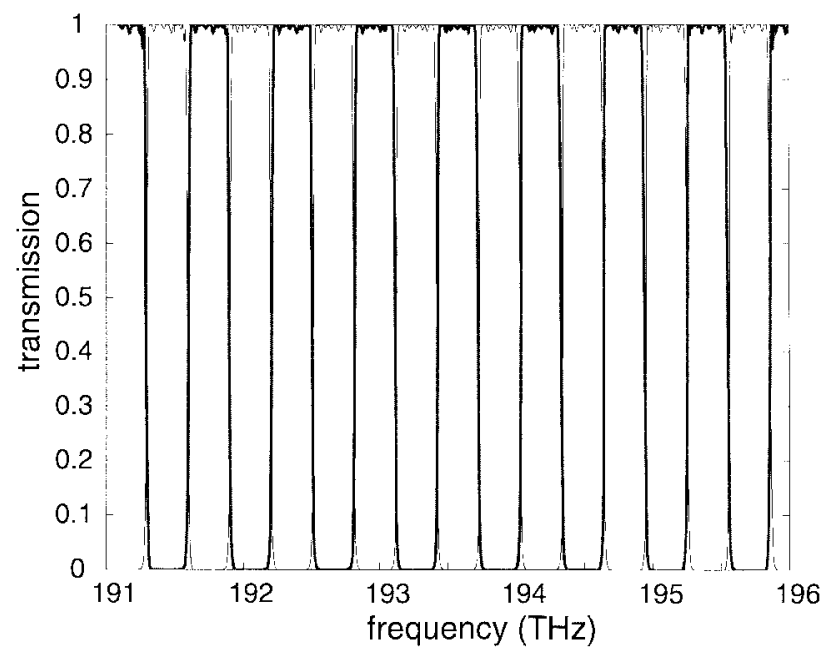

Fig. 9. Simulated result for two filters (thick and thin lines) which are slightly different in periodicity. Over a 40-nm wide region around the $1.55-\mu \mathrm{m}$ center wavelength, eight periods are included. The two filters look "complementary" to each other.

will decrease quickly as the frequency periodicity increases. Compared to the fiber grating technology, the overall size of the proposed structure is very small and has the potential for integrated photonic implementations.

\section{WAVELENGTH SWITCHING USING LOGARITHMIC FILTER CHAINS}

In this section, we discuss how to connect the rectangular shaped periodic filters to achieve logarithmic scaling complexities for WDM applications.

The first and most straightforward way is to connect pairs of complementary transmission filters with $2 \times 2$ switches in series as shown in Fig. 8. As the frequency period $\Delta f$ in the filter transfer function is small compared to the center frequency, a small change in the periodicity would cause a slip of a half period in the frequency range of interest without noticeable change in the period. This results in a complementary filter function in the frequency range of interest. Fig. 9 shows an example of two filters whose transfer functions are complementary. Each filter has eight periods over a 40-nm wide bandwidth. The mirror separation distances in the two 


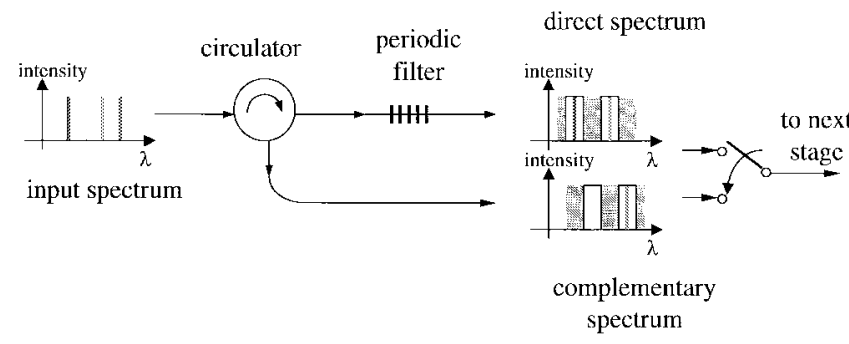

Fig. 10. Obtaining the direct and complementary spectral outputs from the transmitted and reflected filter outputs using an optical circulator.

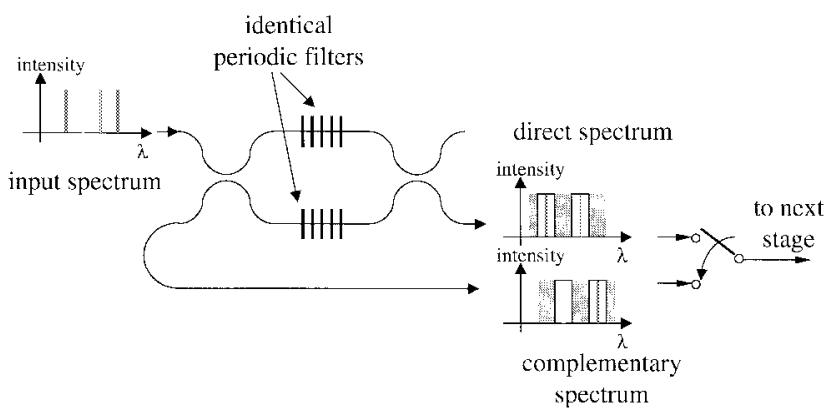

Fig. 11. Obtaining the direct and complementary spectral outputs from a Mach-Zehnder interferometer (MZI). Identical filters are incorporated in two arms of equal length. The direct and complementary outputs propagate in opposite directions.

filters are different from each other by about $1 / 300$ which corresponds to about $0.2 \mu \mathrm{m}$ in length if implemented on silicon.

The second way to obtain the complementary spectral outputs is by using the transmitted and reflected spectrum of the same rectangular shaped filter. The direct spectrum is obtained from the transmitted output. The reflected complementary spectrum can be separated from the input signal by using an optical circulator as shown in Fig. 10.

The third way to obtain the direct and complementary spectra can be achieved by using a Mach-Zehnder configuration with identical filters incorporated in the two arms (Fig. 11). The direct and complementary spectra will propagate in opposite directions. This method has been used with fiber gratings for optical WDM add-drop [16].

As pointed out earlier, to pluck out one out of $N$ wavelengths, only $\log _{2} N$ stages and $\log _{2} N$ switches are required. Electrooptic $2 \times 2$ switches with giga-Hertz switching speeds are available commercially. A fast WDM demultiplexer with $\log _{2} N$ complexity and the capability to filter out any one wavelength at nanosecond speed is therefore possible. Such devices should be good candidates for future dense WDM packet switched optical networks which will have more flexibility and make better sharing and utilization of the enormous capacity provided by the optical fiber.

\section{CONCLUSION}

We have proposed a WDM demultiplexer with $\log _{2} N$ complexity. The key to achieve the $\log _{2} N$ scaling complexity is a series of rectangular shaped periodic frequency filters capable of generating complementary spectral outputs. The direct or complementary spectral output from each filter output is selected by a $2 \times 2$ optical switch as the input to the next stage which has twice the spectral periodicity as the current stage. A design example of the rectangular-shaped periodic filter in the form of a 1-D photonic crystal is given in this paper. The photonic crystal consists of layered dielectric Bragg mirrors of 50\% power reflectivity in the wavelength range of interest. The separation between the mirrors determines the periodicity of the filter in the frequency domain. The number of mirrors determines the sharpness of the band edge. A compact ridged waveguide implementation is proposed for such filters. This kind of filters has very good potential for future integrated photonic implementation.

Apart from its favorable scalability in future dense WDM systems with a large number of wavelengths, very fast electrooptic $2 \times 2$ switches can be incorporated in these $\log _{2} N$ demultiplexers, which will be valuable for packet switched optical data networks where the switching delay is important for high performance.

\section{REFERENCES}

[1] P. E. Green, Fiber Optic Networks, Prentice-Hall, 1993, p. 38.

[2] P. E. Green and L. A. Coldren et al., "All-optical packet-switched metropolitan-area network proposal," J. Lightwave Technol., vol. 11, no. $5 / 6$, pp. $754-763$, May/June 1993

[3] S. R. Mallinson and J. H. Jerman, "Miniature micromachined FabryPerot interferometers in silicon," Electron. Lett., vol. 23, no. 20, pp. 1041-1043, Sept. 1987.

[4] J. Stone and L. W. Stulz, "Pigtailed high-finesse tunable fiber FabryPerot interferometers with large, medium and small free spectral range," Electon. Lett., vol. 23, pp. 781-783, 1987.

[5] A. A. Saleh and J. Stone, "Two-stage Fabry-Perot filters as demultiplexers in optical FDMA LAN's," J. Lightwave Technol., vol. 7, pp. 323-330, Feb. 1989.

[6] J. P. Laude and J. M. Lerner, "Wavelength division multiplexing/demultiplexing (WDM) using diffraction gratings," in Proc. SPIE-Application, Theory and Fabrication of Periodic Structures, vol. 503, 1984, pp. 22-28.

[7] C. Dragone, "An $N \times N$ optical multiplexer using a planar arrangement of two star couplers," IEEE Photon. Technol. Lett., vol. 3, pp. 812-815, Sept. 1991.

[8] R. T. Hofmeister and S. M. Gemelos et al., "Project LEARN-Ligh exchangeable, add/drop ring network," in Proc. OFC'97 post deadline paper, PD25, IEEE.

[9] E. Yablonovitch, "Photonic band-gap structures," J. Opt. Soc. Amer. B, vol. 10, no. 2, pp. 283-295, Feb. 1993.

[10] P. Yeh, Optical Waves in Layered Media. New York: Wiley, 1988, ch 5-6.

[11] A. Mori and Y. Ohishi et al., "1.5 $\mu \mathrm{m}$ broadband amplification by Tellurite-based EDFA's," in Proc. OFC'97, post deadline paper, PD1, IEEE.

[12] J. S. Foresi, P. R. Villeneuve, J. Ferrera, E. R. Thoen, G. Steinmeyer, S. Fan, J. D. Joannopoulus, L. C. Kimerling, H. I. Smith, and E. P. Ippen, "Photonic-bandgap microcavities in optical waveguides," Nature, vol. 390, pp. 143-145, Nov. 13, 1997.

[13] A. Talneau, C. Ougier, and S. Slempkes, "Multiwavelength grating reflectors for widely tunable laser," IEEE Photon. Technol. Lett., vol. 8, pp. 497-499, Apr. 1996.

[14] C. Wei, D. Van Thourhout, I. Moerman, S. Goeman, K. Vandeputte, and R. Baets, "An integrated optical add/drop multiplexer in InP based on elliptic couplers and Bragg grating reflector," in Proc. 1997 Dig. IEEE/LEOS Summer Topical Meetings: WDM Components Technol., paper ThA2, pp. 28-29.

[15] M. C. Oh, M. H. Lee, J. H. Ahn, H. J. Lee, and S. G. Han, "Polymeric wavelength filters with polymer gratings," Appl. Phys. Lett., vol. 72, no. 13, pp. 1559-1561, Mar. 30, 1998.

[16] T. Mizuochi and T. Kitayama, "Interferometric cross talk-free optica add/drop multiplexer using cascaded Mach-Zehnder fiber gratings," in Proc. Tech. Dig. OFC'97, WL14, IEEE, pp. 176-177. 
Cedric F. Lam (S'98) received the B.Eng. degree with first class honors in electrical and electronic engineering from the University of Hong Kong, Hong Kong, in 1993. He is currently working toward the Ph.D. degree in electrical engineeringat the University of California at Los Angeles (UCLA), under the direction of Prof. E. Yablonovitch.

His research interest is in fiber-optic communication and optical CDMA systems. He held the position of teaching fellow at UCLA Electrical Engineering Department from 1996 to 1998 as the instructor of the course Introduction to Unix and $\mathrm{C} / \mathrm{C}^{++}$

Mr. Lam was a recipient of Sir Edward Youde fellowship from 1994 to 1997.

Rutger B. Vrijen was born September 17, 1970, in Groningen, The Netherlands. He received the Ph.D. degree in physics from the University of Amsterdam, The Netherlands, in 1997.

As a graduate student he experimentally studied the physics of atoms in short laser pulses of extremely high intensity. He is currently a postdoctoral fellow with the Electrical Engineering Department at the University of California at Los Angeles (UCLA). His research interests include laser physics, classical and quantum optics, and optoelectronics.

Dr. Vrijen is a member of the Optical Society of America (OSA).

Patty P. L. Chang-Chien received the B.S. degree in electrical engineering from California Institute of Technology, Pasadena, in 1996. She received the M.S. degree in electrical engineering from the University of California at Los Angeles (UCLA), in 1997. She is currently pursuing the Ph.D. degree at University of Michigan, Ann Arbor.

She is currently with the University of Michigan, Ann Arbor, and her interest is in microelectromechanical (MEM) systems.
Daniel F. Sievenpiper (S'98) received the B.S. degree in electrical engineering from the University of California at Los Angeles (UCLA), in 1994. He is currently working toward the Ph.D. degree in electrical engineering, also at UCLA, under the direction of Prof. E. Yablonovitch.

His current research is in the field of photonic bandgap structures, with particular emphasis on metallo-dielectric structures for microwave applications.

Eli Yablonovitch (F'92) received the Ph.D. degree in applied physics from Harvard University, Cambridge, MA, in 1972.

He worked for two years at Bell Telephone Laboratories, and then became a Professor of Applied Physics at Harvard University. In 1979, he joined Exxon to do research on photovoltaic solar energy. Then in 1984, he joined Bell Communications Research, where he was a Distinguished Member of Staff, and also the Director of Solid-State Physics Research. In 1992, he joined the University of California at Los Angeles (UCLA), where he is Professor of Electrical Engineering. His work has covered a broad variety of topics such as nonlinear optics, laser-plasma interaction, infrared laser chemistry, photovoltaic energy conversion, strained quantum-well lasers, and chemical modification of semiconductor surfaces. Currently, his main interests are in the fields of epitaxial liftoff technology, photonic band structure, and optical code division multiplexing.

Dr. Yablonovitch has received the Alfred P. Sloan fellowship (1978-1979), the Adolf Lomb Medal of the Optical Society of America (1978), an R\&D 100 award (1990), the W. Streifer Scientific Achievement award of the IEEE/LEOS (1993), and the R. W. Wood Prize of the Optical Society of America (1996). He chaired the 1979 Gordon Conference on Nonlinear Optics and Lasers. He has been named a Fellow of the American Physical Society (1990) and the Optical Society of America (1982), and is a member of Eta Kappa Nu. 